\title{
Mobile Backhaul in Heterogeneous Network Deployments: Technology Options and Power Consumption
}

\author{
Paolo Monti*, Member, IEEE, Sibel Tombaz, Member, IEEE, \\ Lena Wosinska, Senior Member, IEEE, Jens Zander, Member, IEEE \\ ${ }^{I}$ KTH Royal Institute of Technology, \\ Communication Systems Department, \\ Electrum 229, 16440 Kista, Sweden \\ * Tel: +46(8) 790 4676, Fax: +46(8) 790 4090, e-mail: pmonti@kth.se
}

\begin{abstract}
Mobile communication networks account for $0.5 \%$ of the global energy consumption, a value that is expected to double within the next five years. For this reason, means of reducing the energy consumption in cellular mobile radio networks has recently gained great interest within the research community. In mobile networks the backhaul contribution to the total power consumption is usually neglected because of its limited impact compared to that of the radio base stations. However, meeting the almost exponential increase in mobile data traffic requires a large number of (mainly small) base stations. This means that backhaul networks will take a significant share of the cost and the energy consumption in future systems. Their actual contribution to the energy consumption will depend on the radio base station deployment scenario as well as on the technology and topology choices for the backhaul itself.

This paper presents an initial assessment of the power consumption of two established backhaul technologies, i.e., fiber and microwave. For the microwave case, three backhaul topologies are considered, i.e., tree, ring and star, while for the fiber case only one topology is analysed, i.e., a dedicated point-to-point star. The presented results, assuming off-the-shelf products and based on todays network capacity levels, confirm the importance of considering the backhaul when minimizing the total power consumption in heterogeneous network scenarios. They also show the impact of the basic technology and topology choices of the backhaul for minimizing total power consumption.
\end{abstract}

Keywords: Green networks, mobile backhaul, heterogeneous wireless networks, pico base stations, microwavebased backhaul, fiber-based backhaul.

\section{INTRODUCTION}

Despite the large potential of ICT for reducing carbon emissions in other sectors of society, there is a growing concern for the power consumption of ICT itself. Projections show that by the year 2015 capacity requirements in mobile networks will be a up to 30 times larger than today [1]. How to meet this demand without leading to an unacceptable increase in network power consumption is a topic that currently is gaining a lot of interest not only in academia.

One way of catering for the increasing capacity requirements in mobile networks is the use of heterogeneous network deployment strategies. Heterogeneous networks present an alternative to macro densification where the macro layer is complemented by the deployment of small, low power, Pico/Micro base stations. The key rationale behind a heterogeneous network deployment is to tailor the network deployment to the expected traffic levels, i.e., to provide high capacity only where it is needed. Thereby we reduce the number base stations needed and the energy used. The performance of different deployment schemes in terms of energy efficiency were also studied by means of simulation in [2] and [3].

However, most studies of power consumption in mobile networks omit contributions from the backhaul to the total network power and only consider the aggregated power consumption of the base stations [4][5]. In terms of total network power consumption the backhaul contribution is not necessarily the same for all mobile network deployment scenarios. The authors in [6] have shown that the relative effect of the backhaul power consumption is non-negligible for scenarios with an increasing number of small (low power) base stations. This is due to the large difference in power consumption between base stations of different size whereas the backhaul contribution per base station is similar. This means that the total power consumption of a heterogeneous network deployment to a larger degree is affected by the backhaul architecture. This calls for an understanding of how different architectural options for the backhaul affect the total backhaul power consumption. Thus, in order to obtain consistent and realistic results on the overall network power consumption, so to define strategies for network optimization, an assessment of the various backhaul technologies and their energy consumption should be modelled and incorporated into the analysis.

Currently backhaul for conventional Macro base stations is to a large extent based on microwave, copper and fiber. These technologies are complementary in a sense that they offer different advantages and disadvantages for different deployment scenarios. A fiber-based backhaul comes at a relatively high CAPEX and costly deployment but offers long-term support with respect to increasing capacity requirements. Microwave-based 
backhaul is an attractive choice for meeting backhaul requirements [7] with short time-to-market, low investment in infrastructure and simple deployment [8]. Digital Subscriber Line (DSL) is attractive in the presence of an existing copper infrastructure. Continued improvements in DSL-technology are also enabling DSL to play an important role for mobile backhaul with increasing capacity requirements [9]. In an emerging heterogeneous network scenario, with a large number of small cells that need to be backhauled, an increasing variety of backhaul technologies and solutions is expected, including also new wireless options. Heterogeneous network backhauling leads to new challenges compared to Macro backhauling, which is expected to result in a different technology mix for the backhaul solution. Deployment will depend on several factors, such as, existing infrastructure, spectrum and licence costs, availability of equipment, operator business situation, etc.

This paper presents an analysis of the power consumption for todays heterogeneous network deployment scenarios, including the effect of backhaul for two established backhauling technologies, i.e., fiber, and microwave. The objective is to make an initial assessment of what is the impact on the total network power of the various technology and architectural options for the backhaul, where off-the-shelf products and current traffic levels (i.e., capacity requirements) are used for the assessment. The main differences, potentials and challenges of these technologies are discussed in [7] with a specific focus on cost, however to the best of the authors' knowledge, the power consumption perspective has not been well modelled and investigated. In order to do so first the main characteristics of each backhaul technology will be introduced, and their most common topology options will be analysed. Then two power models, one for the microwave case and one for the fiber case, will be presented and used in a case study to assess the power performance of each technology. The obtained results will then be leveraged to see how different architectural options for a given backhaul technology affect both the total backhaul and the total mobile network power consumption. Simulation results show that already in todays heterogeneous network deployments, from a mere energy consumption perspective, fiber-based backhaul solutions might be preferable due to their relatively low impact in terms of extra power consumption.

\section{MOBILE BACKHAUL ARCHITECTURES}

When it comes to the choice of a particular backhaul technology and topology, there are a number of factors that need to be considered, such as, Capital Expenditures (CAPEX), Operating Expenditures (OPEX), propagation and spectrum conditions, Quality of Service (QoS) requirements (i.e., mostly delay), and requested resiliency levels. This section presents the assumption made for each technology and architectural choice. In this work it is assumed that only two types of base stations are available for a heterogeneous network deployment scenario, i.e., Macro and Pico base stations. Fig. 1 shows the basic architectural backhaul options that are considered and later used for comparison from a power consumption perspective.

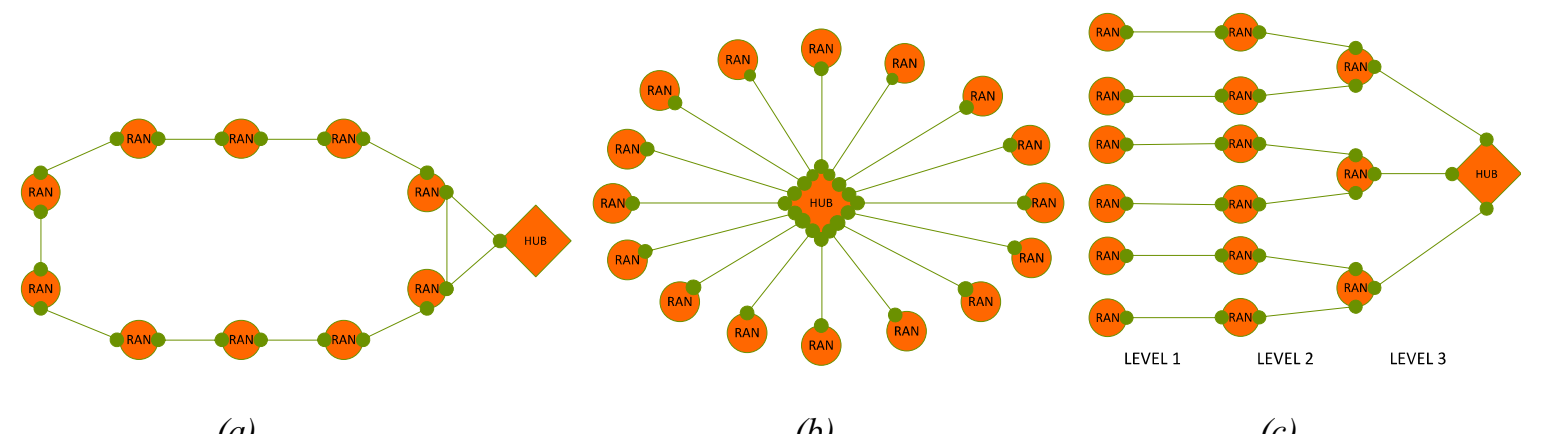

Figure 1: Different backhaul topology solutions [10]: (a) ring topology, (b) star topology, an (c) tree topology. Circles represent Radio Access Network (RAN) sites, while rectangles represent hub nodes.

As can be seen from Fig. 1, each base station, i.e., RAN, site is connected to one or more sites, depending on the topology under consideration. All traffic from the base stations is assumed to be backhauled through a hub node. In this work it is assumed that any base station in the network, regardless of its type (i.e., Macro and/or Pico), can be a hub node. Depending on the total number of base stations in the studied area, and also depending on the backhaul architectural choice (i.e., maximum number of base stations in each topology), more than one level of aggregation can be considered, i.e., multiple hub nodes might be present in an area. Each hub node is connected to the area aggregation point (i.e., a sink node, not shown in the figure) that in turn connects to an aggregation network (i.e., either a metro or core segment). If more than one backhaul link originates or terminates at any base station site, that particular site is assumed to be equipped with a switch.

For the microwave-based backhaul we consider tree, ring and star topologies and these are compared to a star-like fiber topology. The backhaul links are dimensioned for peak rate from the base stations to the hub node. This is more aggressive compared to dimensioning guidelines in [11] since it implies significant overbooking already at the first aggregation stage. Each backhaul link is dimensioned to cater for the capacity requirements of 
the connected base station and of any additional base station backhauled via that base station site (this applies only for the ring and tree case). It should however be noted that despite the effort to dimension the backhaul networks in a similar way for the different topologies there are inherent differences between the topologies with respect to aspects such as latency and resilience.

For star topologies there are separate microwave links from the hub to each site. The drawback with a starlike topology is the limited area that can be covered by a single star due to the reach limitations of the links. To provide coverage via microwave, star topologies require longer LOS (line of sight) links. Star topologies also lead to poor frequency reuse. All links terminate at the same point (i.e., the hub node), and links using the same frequency are more likely to interfere with each other. Rings are advantageous in terms of protection but latency may be an issue. The maximum capacity of a ring will also limit the number of sites that can be served by each ring. A tree topology, on the other hand, is sensitive to faults on the feeder links. In terms of power consumption the star topology can be seen to be the most efficient assuming that a single star can serve the considered area and assuming that the link power is independent of the reach. This is because a star topology requires a minimum of one link per base station site and enables maximum statistical multiplexing at the central hub.

Since fiber is able to provide sufficient reach for the considered area, for the fiber-based backhaul case a single star scenario is assumed. In the considered fiber-based star all base stations are connected to a single central hub node, that works also as the sink towards the aggregation network (more details about this backhaul architecture can be found in [6]). The hub node is equipped with one or more aggregation switches (if the number of the traffic streams to be aggregated so requires).

\section{BACKHAUL POWER CONSUMPTION MODELS}

This section presents power models for mobile networks including the contribution from the backhaul. A characterization of the power consumption of the individual Macro and Pico base stations can for example be found in [3] where the model parameters are derived based on data found in the literature.

In the microwave case the total power consumption of a heterogeneous mobile radio network including the backhaul part can be written as:

$$
P_{t o t}^{M W}=\sum_{i=1}^{m} N_{i} P_{i}+P_{b h}^{M W}
$$

where $m$ is the number of base station types used in the network, $N_{i}$ is the total number of base stations of a specific type $i$ (e.g., Macro or Pico base stations), $P_{i}$ is the power consumption of a base station of type $i$, and $P_{b h}^{M W}$ is the entire backhaul power consumption. $P_{i}$ is modelled as a linear function of the radiated power:

$$
P_{i}=a_{i} P_{t x}+b_{i} .
$$

In equation (2) the parameter $a_{i}$ accounts for the scaling of the base station power consumption with the transmitted power, i.e., the power associated with radio frequency (RF) amplifiers and the feeder losses. The parameter $b_{i}$ is associated with the baseline power that is independent of the transmitted power, i.e. signal processing and site cooling. The backhaul power contribution $P_{b h}^{M W}$ can be modelled as:

$$
P_{b h}^{M W}=P_{\text {sink }}+\sum_{j=1}^{N_{B S}} P_{j}^{M W}
$$

where $P_{\text {sink }}$ represents the power consumption of the area sink node, $N_{B S}$ the total number of base stations (regardless of their type) in the entire area, and $P_{j}^{M W}$ represents the power associated with microwave backhaul operations at the base station site $j$. Assuming that $C_{j}$ represents the total aggregated capacity that base station $j$ has to backhaul, and that $N_{j}^{\text {ant }}$ represents the number of microwave antennas (i.e., number of microwave links) used at base station $j, P_{j}^{M W}$ can be expressed as:

where

$$
P_{j}^{M W}=P_{j, a g g}\left(C_{j}\right)+P_{s w i t c h}\left(N_{j}^{a n t}, C_{i}\right),
$$

$$
P_{j, a g g}\left(C_{j}\right)=\left\{\begin{array}{l}
P_{l o w-c}, \text { if } \mathrm{C}_{j} \leq T h_{\text {low-c }} \\
P_{\text {high-c }}, \text { otherwise }
\end{array} P_{j, \text { switch }}\left(N_{j}^{a n t}, C_{j}\right)=\left\{\begin{array}{l}
0, \text { if } N_{j}^{a n t}=1 \\
P_{S} *\left\lceil\frac{C_{j}}{C_{\text {switch }}^{M A X}}\right\rceil, \text { otherwise }
\end{array} .\right.\right.
$$

According to equation (5) the power consumption for transmitting and receiving the aggregate backhaul traffic via microwave $\left(P_{j, a g g}\right)$ is a step function with respect to the total backhauled capacity $\left(C_{j}\right)$. Two power regions $\left(P_{\text {low-c }}\right.$ and $\left.P_{\text {high-c }}\right)$ for each base station were defined, one for low capacity traffic conditions and the other for 
high traffic capacity conditions. For the sake of simplicity only two capacity regions are considered in this work. The extension beyond two regions is straightforward. Equation (5) is also used to account for the power consumption of the switch $\left(P_{s}\right)$ that needs to be used at any base station site aggregating traffic from more than one base station. The number of switches is a function of the total backhauled capacity and the maximum capacity of a switch $\left(C_{\text {switch }}^{M A X}\right)$. Identical switches are assumed to be used at all base station sites, regardless of the type.

The same reasoning can be applied to compute the value of power consumption of the sink node. $P_{\text {sink }}$ can be expressed as follows:

$$
P_{\text {sink }}=P_{\text {sink }, a g g}\left(C_{\text {sink }}\right)+P_{\text {sink }, \text { switch }}\left(N_{\text {sink }}^{\text {ant }}, C_{\text {sink }}\right),
$$

where

$$
P_{\text {sink }, \text { agg }}\left(C_{\text {sink }}\right)=\left\{\begin{array}{l}
P_{\text {low }-c}, \text { if } \mathrm{C}_{\text {sink }} \leq T h_{\text {low }-c} \\
P_{\text {high }-c}, \text { otherwise }
\end{array} P_{\text {sink }, \text { switch }}\left(N_{\text {sink }}^{\text {ant }}, C_{\text {sink }}\right)=\left\{\begin{array}{l}
0, \text { if } N_{\text {sink }}^{\text {ant }}=1 \\
P_{S} *\left\lceil\frac{C_{\text {sink }}}{C_{\text {switch }}^{M A X}}\right\rceil, \text { otherwise }
\end{array} .\right.\right.
$$

For the fiber-based case the total power consumption of a heterogeneous mobile radio network including the mobile backhaul can be written as:

$$
P_{t o t}^{F I B}=\sum_{i=1}^{m} N_{i} P_{i}+P_{b h}^{F I B}
$$

where according to the parameters defined in [6]:

and

$$
P_{i}=a_{i} P_{t x}+b_{i}+c_{i},
$$

$$
P_{b h}^{F I B}=\left\lceil\frac{1}{\max _{d l}}\left(\sum_{i=1}^{m} N_{i}\right)\right\rceil P_{s}+\left(\sum_{i=1}^{m} N_{i}\right) P_{d l}+N_{u l} P_{u l} .
$$

The parameters $m, N_{i}, a_{i}, b_{i}$, and $P_{s}$ are the same as in the microwave case. On the other hand $c_{i}$ represents the power consumption of a small-form factor pluggable (SFP) optical interface (used to connect a base station to the aggregation switch at the hub node), and $\max _{d l}$ is the maximum number of downlink interfaces available at one aggregation switch. This parameter is used to compute the total number of switches that are needed to collect the backhauled traffic of the mobile network. On the other hand, $P_{d l}$ is the power consumed by one interface in the aggregation switch (one for each backhauled base station), while $N_{u l}$, and $P_{u l}$ are the total number of uplink interfaces, and the power consumption of one uplink interface, respectively. These last two parameters account for the power consumption of the interfaces used to aggregate the backhauled traffic towards a metro/core segment. A more detailed explanation of these parameters can be found in [6].

\section{POWER CONSUMPTION COMPARISONS}

As a basis for comparing the various backhaul architectures we assume two different base station deployment scenarios, each one providing the same area throughput and coverage. The first scenario considers a Macro deployment where capacity is increased by a densification of the Macro base station sites. The second scenario considers a heterogeneous network deployment where capacity is increased by the deployment of Pico cells. The underlying system model and the assumptions used for the network deployment are made in accordance with the work presented in [12]. In each scenario the number of base stations for the Dense Macro and the Macro + Pico cases are determined sequentially until the target area throughput is satisfied.

Table 1. Power consumption parameters, microwave backhaul case [3][13].

\begin{tabular}{|c|c|c|c|c|c|c|c|c|}
\hline Base station type & $a_{i}$ & $b_{i}$ & $P_{t x}[\mathrm{dBm}]$ & $P_{\text {low-c }}[\mathrm{W}]$ & $P_{\text {high }-c}[\mathrm{~W}]$ & $T h_{\text {low-c }}[\mathrm{Mbps}]$ & $P_{s}[\mathrm{~W}]$ & $C_{s w i t c h}^{M A X}[\mathrm{Gbps}]$ \\
\hline Macro & 21.45 & 354.44 & 43 & 37 & 92.5 & 500 & 53 & 36 \\
\hline Pico & 5.5 & 38 & 30 & 37 & 92.5 & 500 & 53 & 36 \\
\hline
\end{tabular}

In this work the base stations are assumed to be of 3G Universal Mobile Telecommunications System (UMTS) type each with a peak user downstream data rate of $100 \mathrm{Mbps}$ in total. Macro base stations are assumed to have 3 -sectors with antennas above rooftop for longer range. They have a maximum of three carriers per sector. On the other hand, Pico base stations have single carrier and omni-directional antennas. The area considered is a square region of four by four kilometers. For the microwave case the power consumption parameters of each type of base station used in the comparison are summarized in Table 1, while Table 2 summarizes the power consumption parameters for the fiber backhaul case. Results presented in this section are for the ring and star topologies shown in Fig. 1, where the number of nodes is varied as part of the assessment. For simplicity, in the 
tree topology case, a complete binary tree is assumed (compared to the variant shown in Fig. 1). The exact structure of the tree is not expected to affect the general validity of the conclusions that will be drawn from the results.

Table 2. Power consumption parameters, fiber backhaul case [3][6].

\begin{tabular}{|c|c|c|c|c|c|c|c|c|}
\hline Base station type & $a_{i}$ & $b_{i}$ & $c_{i}[\mathrm{~W}]$ & $P_{d l}[\mathrm{~W}]$ & $P_{u l}[\mathrm{~W}]$ & $\max _{d l}$ & $P_{s}[\mathrm{~W}]$ & $C_{\text {switch }}^{M A X}[\mathrm{Gbps}]$ \\
\hline Macro & 21.45 & 354.44 & 1 & 1 & 2 & 24 & 300 & 24 \\
\hline Pico & 5.5 & 38 & 1 & 1 & 2 & 24 & 300 & 24 \\
\hline
\end{tabular}

Tables 3 and 4 show, for the microwave case, the power consumption associated with backhaul operations, i.e., $P_{b h}^{M W}$ defined in section 3, as a function of the area throughput requirements. For all three cases (i.e., tree, star and ring) the value $P_{b h}^{M W}$ is normalized using the value of the total area covered, i.e., $P_{b h}^{M W} / 16 \mathrm{~km}^{2}$.

Table 3. Value of $P_{b h}^{M W}[W]$ normalized by the total area covered: Dense Macro case.

\begin{tabular}{|c|c|c|c|c|c|c|c|c|c|c|c|c|}
\hline \multirow{3}{*}{$\begin{array}{c}\text { Area } \\
\text { Throughput } \\
\text { [Mbps/km2] }\end{array}$} & \multicolumn{4}{|c|}{ Tree } & \multicolumn{4}{|c|}{ Star } & \multicolumn{4}{|c|}{ Ring } \\
\hline & \multicolumn{4}{|c|}{ Max \# hops } & \multicolumn{4}{|c|}{ Max \# nodes } & \multicolumn{4}{|c|}{ Max \# nodes } \\
\hline & 2 & 3 & 4 & 5 & 6 & 8 & 12 & 16 & 4 & 6 & 8 & 10 \\
\hline 40 & 103.3 & 122.9 & 126.4 & 126.4 & 87.1 & 80.0 & 76.4 & 76.4 & 145.6 & 208.5 & 218.0 & 222 \\
\hline 60 & 194.9 & 218.1 & 221.6 & 242.6 & 157.0 & 146.3 & 135.7 & 132.1 & 270.3 & 416.0 & 422.9 & 423 \\
\hline 80 & 277.0 & 301.8 & 312.4 & 312.4 & 224.5 & 210.3 & 192.5 & 185.4 & 389.0 & 607.6 & 607.6 & 611.1 \\
\hline
\end{tabular}

Based on the results it is possible to draw some conclusions on the topology impact on power consumption. As expected the value of $P_{b h}^{M W}$ increases with the capacity requirements which affects the numbers of base station deployed in the network. Among the three cases, the ring-based backhaul solution is the most costly in terms of power. This is due mainly to two factors. Firstly, each base station needs to be equipped with a switch to support the ring structure. Secondly, in the considered scenarios the backhaul links always work in the high traffic capacity region due to a large amount of pass-through traffic. The ring structure, on the other hand is able to handle single link failures, compared to the star topology case, which is the least power consuming among the three topology cases. This highlights the important trade off between power consumption and resiliency. In the star case, the only base station equipped with a switch and working in the high traffic capacity region is the hub node. The tree topology case, can in terms of power consumption, be seen as an intermediate case where a few nodes are required to operate in the high traffic region. Going towards a heterogeneous network deployment scenario, we see that the backhaul has a non-negligible impact on the power consumption. This is due to the increased number of base stations that need to be backhauled. All three topology cases experience a power consumption increase of at least $100 \%$ (for the considered backhaul segment) compared to the Dense Macro scenario.

Table 4. Value of $P_{b h}^{M W}[W]$ normalized by the total area covered: Macro + Pico case.

\begin{tabular}{|c|c|c|c|c|c|c|c|c|c|c|c|c|}
\hline \multirow{3}{*}{$\begin{array}{c}\text { Area } \\
\text { Throughput } \\
{[\mathrm{Mbps} / \mathrm{km} 2]}\end{array}$} & \multirow{2}{*}{\multicolumn{4}{|c|}{$\frac{\text { Tree }}{\text { Max \# hops }}$}} & \multicolumn{4}{|c|}{ Star } & \multicolumn{4}{|c|}{ Ring } \\
\hline & & & & & & $\operatorname{Max} \neq$ & nodes & & & $\operatorname{Max} \neq$ & nodes & \\
\hline & 2 & 3 & 4 & 5 & 6 & 8 & 12 & 16 & 4 & 6 & 8 & 10 \\
\hline 40 & 264.2 & 290.9 & 301.5 & 308.6 & 208.2 & 197.5 & 183.3 & 176.2 & 365.3 & 559.6 & 573.5 & 556.1 \\
\hline 60 & 582.4 & 641.0 & 662.1 & 672.6 & 457.3 & 425.3 & 393.5 & 375.7 & 804.7 & 1226.1 & 1269.5 & 1269.5 \\
\hline 80 & 852.5 & 925.1 & 956.8 & 958.5 & 658.6 & 616.1 & 566.4 & 545.1 & 1166.9 & 1843.3 & 1843.3 & 1843.3 \\
\hline
\end{tabular}

Fig. 2 provides a comparison of the normalized power consumptions of the backhaul segment for both a microwave-based and a fiber-based backhaul, assuming a heterogeneous network deployment scenario, i.e., the Macro + Pico case. Two extreme cases in terms of topology size, for each microwave topology option, are depicted to demonstrate the relative difference in terms of power consumption performance between fiber and microwave technologies. For small size topologies 2, 6, and 4 nodes are considered for the tree, star, and ring topologies, respectively. On the other hand, large size topologies for trees, stars, and rings are characterized by 5 , 16 and 10 nodes, respectively. The figure shows that a fiber-based backhaul consumes less power regardless of the considered topology, and topology parameters. Hence, from a pure energy consumption perspective the single star fiber-based solution where each base station in the area has a dedicated point-to-point connection with the sink node is preferable. Obviously, such considerations need to be weighted against other important factors such a deployment costs. 


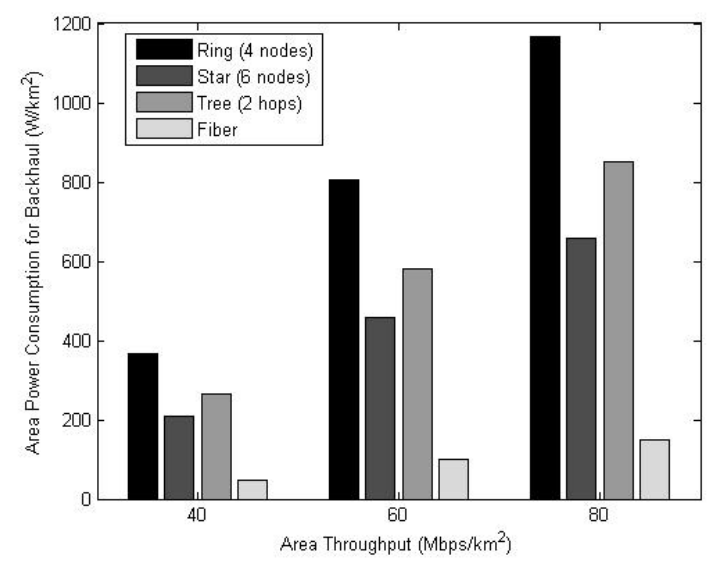

(a)

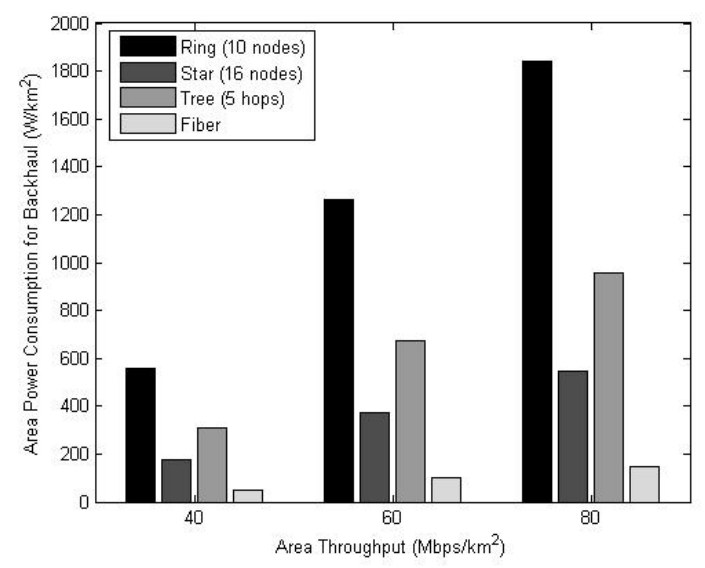

(b)

Figure 2. Area power consumption for the backhaul: microwave vs. fiber in a Macro + Pico case. Two scenarios are considered: small size (a), and (b) large size microwave topologies.

Fig. 3 shows the impact of the power consumption of the backhaul segment on the overall network power consumption. The value of the total network power consumption is shown as a function of the area throughput requirements. Both technology options, i.e., fiber and microwave (with all three topologies options) are considered. The figure confirms the intuition that the impact of the backhaul power consumption is larger for a heterogeneous network scenario compared to dense Macro. This is true regardless of the technology choice for the backhaul. This means that technology and topology considerations for the backhaul will be increasingly important for optimizing the total network power in a heterogeneous network scenario. On the other hand, even with the inclusion of the extra power consumed by the backhaul segment, the use of low power Pico base station still remains the best option to have a network with an overall reduced power consumption, i.e., the power benefits of using a heterogeneous deployment still hold.

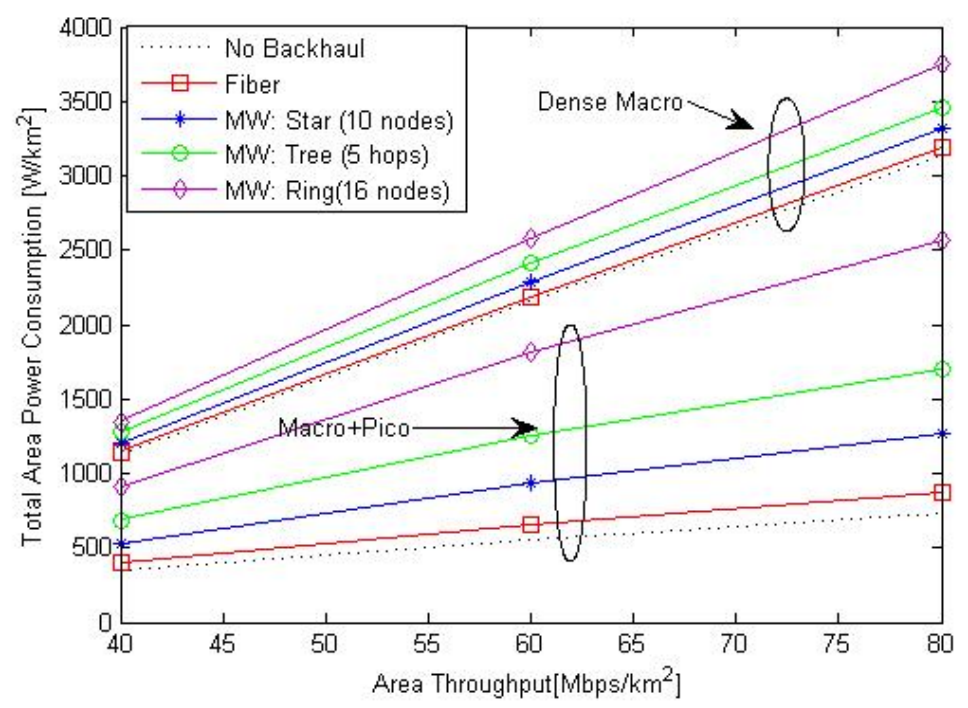

Figure 3. Total area power consumption. Three scenarios are considered, no backhaul, microwave-based backhaul, and fiber-based backhaul.

\section{CONCLUSIONS}

This paper presented a study on the impact of various backhaul options on the overall power consumption of a heterogeneous mobile network. Two backhaul technologies are considered, microwave- and fiber-based. Various topological choices were analysed (assuming off-the-shelf products and current network capacity requirements) and their respective power consumption models were derived. The presented results confirm that the power consumption of the backhaul segment is an important part of the total network power consumption. For this reason the backhaul needs to be carefully included in any deployment strategy with objective of minimizing total network power consumption. Furthermore, results also show that from a pure power consumption perspective a 
star shaped fiber-based topology is preferable. This finding must be weighted against other important factors such a deployment costs, which would be interesting to address in a continuation of this work.

\section{ACKNOWLEDGEMENTS}

The authors would like to thanks to Klas Johansson for sharing his simulation results and Björn Skubic for his valuable technical conversations and input. The work presented in this paper was carried out with the support of the Energy-efficient Wireless Networking (eWIN) and Optical Networking System (ONS) focus projects, part of ICT - The Next Generation (TNG) Strategic Research Area (SRA) initiative at the Royal Institute of Technology.

\section{REFERENCES}

[1] “Cisco visual networking index: Global mobile data traffic forecast update, 2010-2015," Technical Report, Cisco, 2010.

[2] S. Tombaz, M.Usman, and J. Zander, "Energy efficiency improvements through heterogeneous networks in diverse traffic distribution scenarios," in Proc. CHINACOM, Harbin, China, August 2011.

[3] F. Richter, et al., "Energy efficiency aspects of base station deployment strategies for cellular networks," in Proc. IEEE VTC, Anchorage, USA, Sep. 2009.

[4] F. Richter, et al., "Traffic demand and energy efficiency in heterogeneous cellular mobile radio networks," in Proc. IEEE VTC Spring, Taipei, Taiwan, May 2010.

[5] K. Dufkova, et al, "Energy savings for cellular network with evaluation of impact on data traffic performance," in Proc. European Wireless Conference, Lucca, Italy, April 2010.

[6] S. Tombaz, et al., "Impact of Backhauling Power Consumption on the Deployment of Heterogeneous Mobile Networks," in Proc. IEEE GLOBECOM, 2011.

[7] O. Tipmongkolsilp, S. Zaghloul, and A. Jukan, "The evolution of cellular backhaul technologies: Current issues and future trends," IEEE Communications Surveys Tutorials, vol. 13, no. 1, pp. 97-113, 2011.

[8] "High speed technologies for mobile backhaul," White Paper, Ericsson, Sep. 2008.

[9] “Leveraging VDSL2 for mobile backhaul," White Paper, Alcatel Lucent, Sep. 2010.

[10] M. Paoloni, "Crucial economics for mobile data backhaul," White paper, Senza Fili Consulting, 2011.

[11] "Guidelines for LTE Backhaul Traffic Estimation," White Paper, NGMN Alliance, 2011.

[12] K. Johansson, "Cost effective deployment strategies for heterogeneous wireless networks," Ph.D. dissertation, Sweden, November, 2007, KTH Information and Communication Technology.

[13] "Ericsson Microwave Networks portfolio," available at http://www.ericsson.com/ourportfolio/products/microwave-networks 